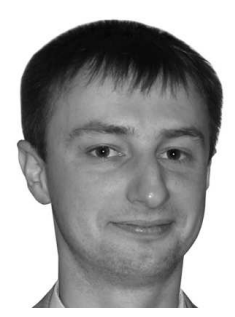

ЗОЛОТОВ

Иван Анатольевич

(Калининградский

государственный технический университет)

\section{ZOLOTOV}

Ivan Anatol'evich

(Kaliningrad, Russian

Federation, Kaliningrad

State Technical University)

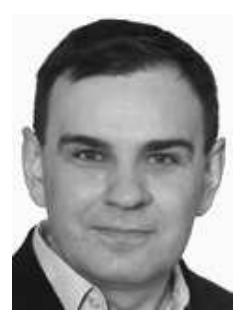

ШАРКОВ

Олег Васильевич

(Калининградский

государственный технический университет)

SHARKOV

Oleg Vasil'evich

(Kaliningrad, Russian

Federation, Kaliningrad

State Technical University)

\section{Анализ напряженно- деформированного состояния внешней обоймы роликовых механизмов свободного хода}

УДК 621.837.2: 539.3

Внешняя обойма - важный конструктивный элемент роликовых механизмов свободного хода (МСX), исследованию напряженно-деформированного состояния которого не уделяют должного внимания. В научной литературе расчет внешней обоймы рекомендуют проводить с учетом значительных допущений по приближенным формулам. В статье с применением компьютерного моделирования и расчета методом конечных элементов проведено исследование распределения деформаций и напряжений во внешней обойме роликовых МСX. Определено, ито наибольшие напряжения возникают в зонах сопряжения пластины с оболочкой и ступицей внешней обоймы, а наибольшие деформации - на свободном крае иилиндрической оболочки. Установлено, что изменение числа роликов от трех до шести приводит к увеличению нагрузочной способности роликовых МСX и уменьшению напряжений и деформаций во внешней обойме.

Результаты вычислений методом конечных элементов подтверждаются теоретическими расчетами по известным формулам. Результаты исследований можно применять для выбора оптимальных геометрических параметров внешней обоймы роликовых МСX.

Ключевые слова: роликовый механизм свободного хода, метод конечных элементов, напряженно-деформированное состояние.

\title{
Analysis of the stress-strain state of an external roller freewheel mechanism cage
}

\section{I.A. Zolotov, O.V. Sharkov}

The stress-strain state of the external cage that is an important structural element of a roller freewheel mechanism (RFM) has not been adequately studied recently. In the scientific literature, external cages are preferably analyzed using simplifying assumptions and approximate formulas. This paper presents the results of computer simulation of strains and stresses in an external RFM cage by the finite element method. It has been found that maximum stresses occur around the joints between a plate and the hub and shell of the external cage. At the same time, maximum deformations occur on the free edge of a cylindrical shell. The study proved that the load capacity of RFMs with six rollers is larger than that for RFMs with three rollers, while the stresses and strains in the external cage are smaller. The results obtained by the finite element method are 
confirmed by theoretical calculations based on well-known formulas. The results of this study can be used for choosing optimum geometrical parameters of external RFM cages.

Keywords: roller freewheel mechanism, finite element method, stress-strain state.

Роликовые механизмы свободного хода (MCX), благодаря важным преимуществам, являются наиболее широко используемым типом механизмов в приводах различных машин. Их основные преимущества: бесшумность в работе; практически мгновенное заклинивание и расклинивание; простота обслуживания и эксплуатации; высокие скорости свободного хода; способность гасить крутильные колебания [1-6].

При этом они, как и все механизмы, осуществляющие рабочий процесс силами трения, имеют весьма существенный недостаток - возникновение значительных нормальных сил. Поскольку коэффициент трения для стальных приработавшихся поверхностей в условиях смазки равен приблизительно 0,04-0,06 [5-7], то возникают нормальные силы, которые в 16-25 раз превышают силы трения, участвующие в передаче нагрузки, и приводят к существенному увеличению напряжений и деформаций в рабочих элементах роликовых МСX.

В роликовых MCX наибольшие напряжения возникают на поверхностях контакта заклинивающихся роликов со звездочкой. Напряжения на поверхностях контакта роликов с внешней обоймой несколько меньше по значению, приблизительно на $15 . .20 \%$. Кроме того, нагруженность звездочки моментами от возникающих сил выше по сравнению с внешней обоймой [8]. Поэтому при расчете роликовых МСX обычно ограничиваются проверкой на прочность по допускаемым касательным или нормальным напряжениям в месте контакта ролика и звездочки $[1-3,9,10]$.

Расчету собственно напряженно-деформированного состояния (НДС) внешней обоймы не уделяют должного внимания. Ее расчетную схему сводят к кольцу, нагруженному сосредоточенными силами, действующими в его плоскости, и используют формулы, предназначенные для расчета колец простой формы с равномер- ным распределением напряжений по сечениям в условиях статического нагружения [1-2].

Такой подход не вполне оправдан, так как разрушение внешней обоймы на практике наблюдается достаточно часто. Например, согласно рекламациям от автопредприятий и эксплуатационным исследованиям в качестве одной из основных причин потери работоспособности приводов стартеров с роликовыми MCX является разрушение их внешних обойм [10]. При этом масса внешней обоймы может составлять 36...50\% общей массы роликовых МСX и обоснованный выбор геометрических характеристик обоймы на основе анализа ее НДС является важным резервом экономии материала.

Анализ НДС таких деталей как внешняя обойма наиболее эффективно выполнять на базе трехмерного твердотельного моделирования методом конечных элементов.

Анализ НДС внешней обоймы роликовых MCX проводился в пакете конечно-элементных расчетов T-FLEX Анализ, функционирующий в среде T-FLEX CAD 3D. В качестве конечного элемента использован тетраэдальный элемент объемного НДС с четырьмя узлами, позволяющий наиболее точно аппроксимировать расчетную модель и обеспечивающий наилучшие показатели с точки зрения временных затрат.

Расчетную схему внешней обоймы роликовых MCX можно представить как короткую тонкостенную цилиндрическую оболочку, свободную на одном крае и сопряженную с кольцевой пластиной, переходящей в цилиндрическую толстостенную оболочку (ступицу), на другом (рис. 1).

Напряженно-деформированное состояние внешней обоймы определяется комплексом геометрических параметров: $D-$ внутреннего диаметра обоймы; $\beta$ - углов расположения роликов, зависящих от их числа $z ; h_{1} / D-$ отношения толщины оболочки $h_{1}$ к внутреннему диаметру обоймы $D ; h_{2} / D-$ отношения толщины пластины $h_{2}$ к диаметру $D ; l / D-$ отношения длины оболочки $l$ к диаметру $D$.

Граничные условия взаимодействия рабочих поверхностей внешней обоймы и роликов задавали в силах. В процессе работы механизма 

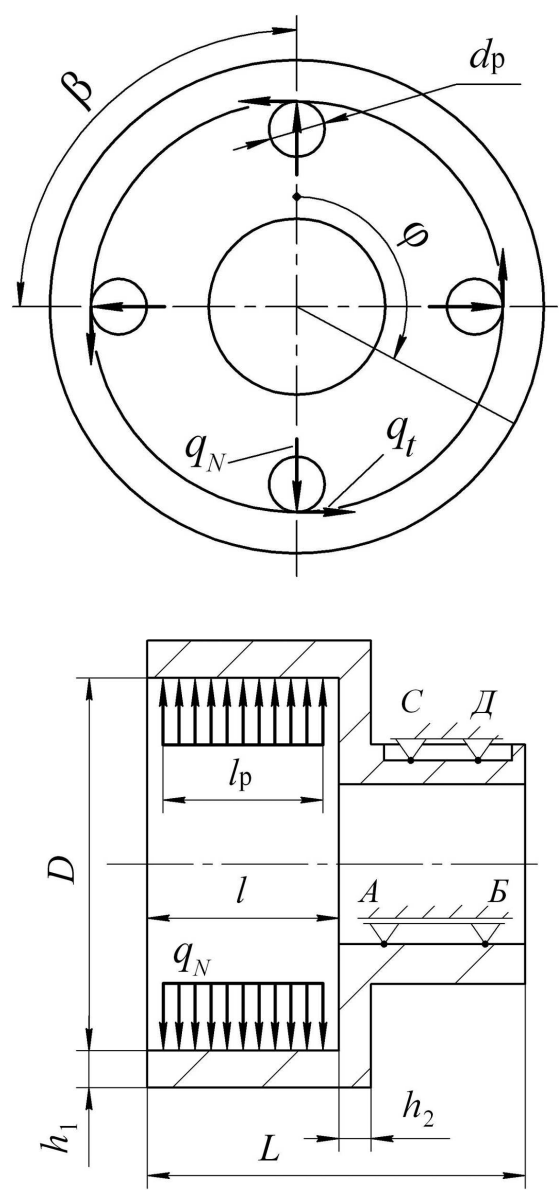

Puc. 1. Расчетная схема внешней обоймы роликовых $\mathrm{MCX}$

на внутреннюю рабочую поверхность внешней обоймы со стороны роликов действуют нормальные и касательные нагрузки, распределенные по их длине.

Моделирование силового взаимодействия рабочих поверхностей внешней обоймы и роликов проводилось путем комбинации силовых факторов - нормальной $q_{N}$ и касательной $q_{t}$ распределенных нагрузок.

Нормальную и касательную нагрузки рассчитывали по формулам

$$
q_{N}=F_{N} / l_{\mathrm{p}} ; \quad q_{t}=q_{N} f,
$$

где $F_{N}$ - нормальная сила, действующая на поверхности контакта ролика с внешней обоймой; $l_{\mathrm{p}}$ - длина ролика; $f$ - коэффициент трения на поверхности контакта ролика с внешней обоймой.

Нормальную силу определяли по условию контактной прочности роликовых МСX по формуле [9]

$$
F_{N}=\frac{l_{\mathrm{p}} d_{\mathrm{p}}\left[\sigma_{H}\right]^{2}}{0,349 E_{\text {пр }}},
$$

где $d_{\mathrm{p}}-$ диаметр ролика; $E_{\text {пр }}-$ приведенный модуль упругости материала; $\left[\sigma_{H}\right]-$ допускаемое контактное напряжение.

Введем следующие коэффициенты: $\psi_{l}=l_{\mathrm{p}} / d_{\mathrm{p}}-$ коэффициент длины ролика, $\psi_{d}=D / d_{\mathrm{p}}-$ коэффициент диаметра ролика. Рекомендуется принимать эти коэффициенты [1] в следующих пределах: $\psi_{l}=1 \ldots 4$, $\psi_{d}=5 \ldots 9[1,2]$. Тогда формулу (1) можно представить в следующем виде:

$$
F_{N}=\frac{\psi_{l}}{0,349 E_{\text {пр }}}\left(\frac{D\left[\sigma_{H}\right]}{\psi_{d}}\right)^{2} .
$$

Граничные условия на контактирующих поверхностях ступицы задавали в перемещениях: распределенную подвижную опору $A D$ на ее внутренней цилиндрической поверхности и распределенную неподвижную СД на поверхностях шпоночного паза (см. рис. 1).

В качестве исследуемых факторов принимали эквивалентное напряжение $\sigma_{e}$ по гипотезе энергии формоизменения (гипотеза Губера-Мизеса-Генки) и радиальную деформацию $\delta_{R}$ внешней обоймы. В качестве независимых факторов использовали геометрические параметры внешней обоймы и величину передаваемой нагрузки.

Исследования проводили для внешней обоймы с геометрическими параметрами: $D=140$ мм; $h_{1} / D=0,1 ; h_{2} / D=0,08 ; l / D=0,35$; общая длина обоймы $L=114$ мм. Ступица внешней обоймы выполнена с внешним шпоночным пазом $b \times h \times l=10 \times 8 \times 40 \quad$ (ГОСТ $\left.23360-78^{*}\right)$.

Число роликов принимали $z=3,4$ и 5, что соответствует диапазону их изменения в отечественных конструкциях роликовых МСХ по ОСТ 27-60-721-84 при значениях коэффициентов $\psi_{l}=2,25$ и $\psi_{d}=7$.

Материал обоймы сталь ШХ15 (модули упругости и сдвига материала $E=2,11 \cdot 10^{5}$ МПа и $G=8,0 \cdot 10^{4}$ МПа, коэффициент Пуассона $\mathrm{v}=0,3)$.

Значение нормальной силы рассчитывали по формуле (2), принимая допускаемое кон- 
тактное напряжение $\left[\sigma_{H}\right]=1500$ МПа $[9,11]$, что соответствует передаваемому роликовым MCX вращающему моменту $T=290 \ldots 480 \mathrm{H} \cdot \mathrm{M}$ при числе роликов 3-5.

Результаты экспериментов представлены в виде кривых, полученных с использованием кубической сплайн-интерполяции данных (оператор «cspline» пакета MathCAD14).

Анализ распределения эквивалентных напряжений по длине внешней обоймы $L$ при различном числе роликов $z$ (рис. 2) показывает, что наибольшие напряжения возникают на свободном крае цилиндрической оболочки $\left(\sigma_{e}=115 \ldots 146 \mathrm{MПа}\right)$, а также в зонах сопряжения пластины с оболочкой и ступицей $\left(\sigma_{e}=155 \ldots 174\right.$ МПа). Характер изменения напряжений подобен при любом числе роликов.

По длине ступицы наблюдается снижение напряжений до 16...20 МПа. Это дает возможность оценивать напряженное состояние только цилиндрической оболочки и части ступицы, которые соответствуют длине $L=80$ мм.

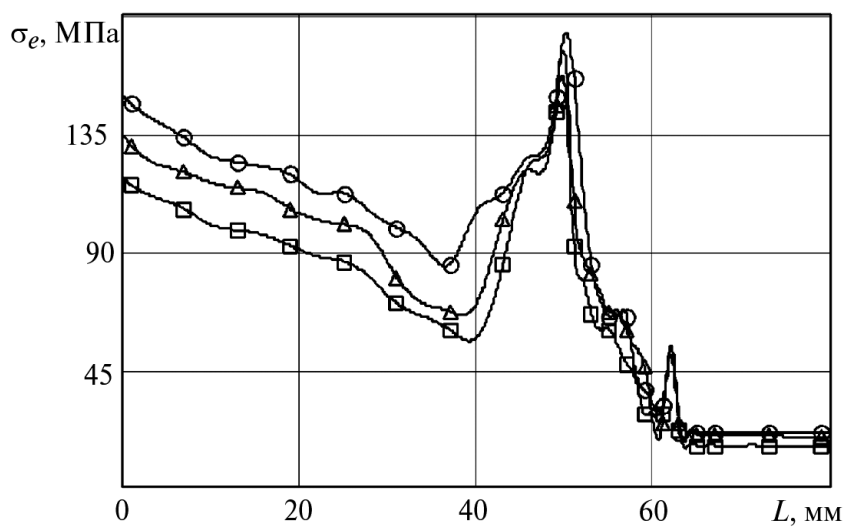

Puc. 2. Зависимость напряжений от длины в осевом сечении внешней обоймы при числе роликов: $\bigcirc-z=3 ; \triangle-z=4 ; \square-z=5$

Анализ распределения эквивалентных напряжений в радиальном сечении внешней обоймы в зависимости от полярного угла $\varphi$ при различном числе роликов $z$ (рис. 3) показывает, что максимальные напряжения $\left(\sigma_{e}=155 \ldots 174\right.$ МПа) наблюдаются в точке приложения нормальной нагрузки под углом $\varphi=180^{\circ}$ (противоположной шпоночному пазу). Другие зоны возникновения пиковых напряжений, которые несколько меньше по величине $\sigma_{e}=82 \ldots 126$ МПа, также соответствуют точкам приложения нормальных сил.

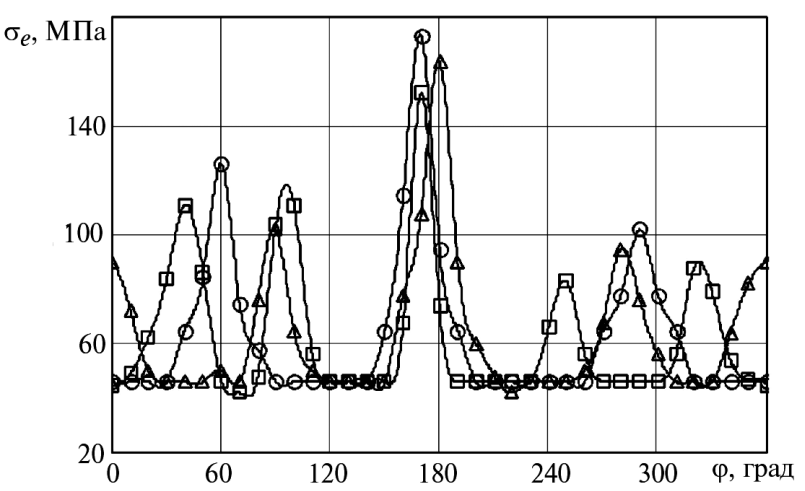

Puc. 3. Зависимость напряжений от полярного угла в радиальном сечении внешней обоймы при числе роликов:

$\bigcirc-z=3 ; \triangle-z=4 ; \square-z=5$

Показанное радиальное сечение внешней обоймы соответствует зоне действия наибольших напряжений в месте сопряжения пластины со ступицей.

Анализ влияния числа роликов на напряженное состояние внешней обоймы устанавливает парадоксальное явление - увеличение числа роликов с 3 до 5, а, следовательно, суммарной нагрузки на обойму, приводит к уменьшению возникающих в ней напряжений в $1,12-1,26$ раза.

Для подтверждения этой закономерности проведем сравнительный расчет влияния роликов на НДС внешней обоймы по другим известным методикам. Используем широко известную формулу для расчета внешней обоймы роликовых МСX [2], которую с учетом принятых обозначений можно представить в следуюшем виде:

$$
\sigma_{e}=\frac{F_{N}}{4 h_{1} l}\left(\frac{1}{\sin (\pi / z)}+\frac{1,5 \pi\left(D+h_{1}\right)}{h_{1} z}\right) .
$$

Число роликов может изменяться в весьма широких пределах от 3 до 15 и более в зависимости от функционального назначения МСX [2, 7]. Например, в отечественных роликовых MCX по ОСТ 27-60-721-84 применяют 3 или 5 роликов, в зарубежных конструкциях МСX фирм GMN, Stieber, (Германия), Renold (Великобритания) число роликов составляет от 6 до 14. Расчет по формуле (3) проводили для наиболее часто встречающего в практике диапазона изменения числа роликов $z=3-6$. 
Характер изменения результирующего (эквивалентного) напряжения от числа роликов, полученный на основании расчетов по формуле (3) и МКЭ с принятыми величинами геометрических и нагрузочных параметров внешней обоймы, показан на рис. 4.

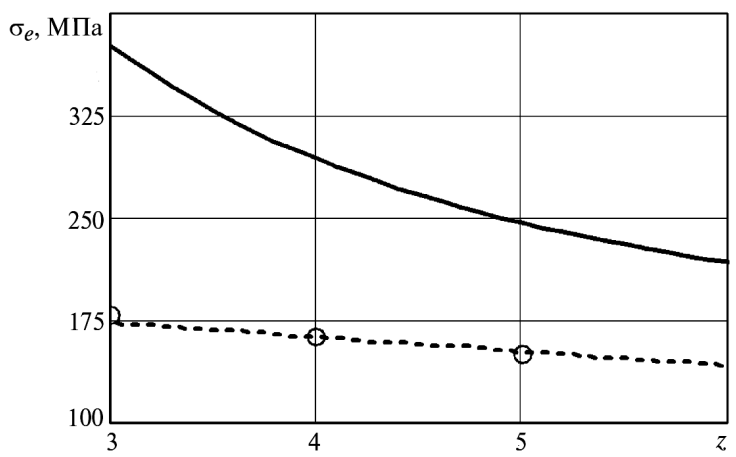

Pис. 4. Зависимость напряжений от числа роликов: - - - - теоретический расчет; ---- - расчет МКЭ

Полученные результаты подтверждают, что увеличение числа роликов в указанном диапазоне приводит к уменьшению значений возникающих напряжений. По-видимому, это можно объяснить следующим. Рассмотренная конструкция внешней обоймы является 6 раз статически неопределимой системой, в которой при увеличении числа точек приложения нагрузки может происходить частичная компенсация (с учетом знака) возникающих напряжений. Подобное явление встречается при расчете изгибной прочности зубьев, когда на растянутой стороне зуба результирующее напряжение по величине не только меньше суммы составляющих его напряжений, но и одного из них, как правило, напряжения изгиба [9].

Следует также отметить, что расчет по формуле (3) дает завышенное в 1,52-2,17 раза значение напряжения, возникающего в обойме, в сравнении с расчетом МКЭ.

Анализ распределения радиальной деформации по длине внешней обоймы при различном числе роликов $z$ (рис. 5) показывает, что наибольшие деформации $\left(\delta_{R}=34 . . .73\right.$ мкм) возникают на свободном крае цилиндрической оболочки.

Затем деформации постепенно уменьшаются по длине цилиндрической оболочки до значения $\delta_{R}=12 \ldots 16$ мкм и в зоне ступицы составляют не более $\delta_{R}=5$ мкм. Характер измене-

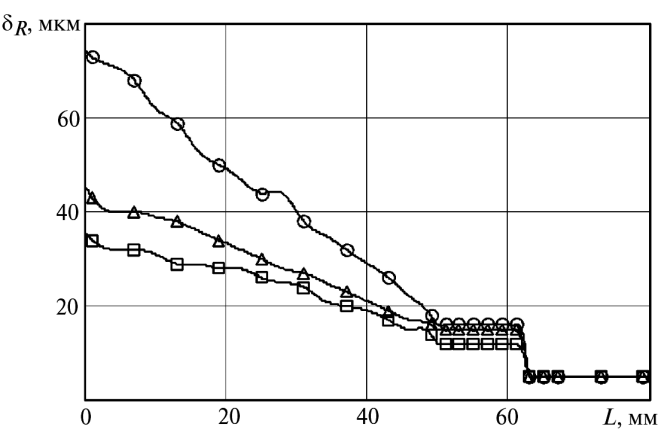

Puc. 5. Зависимость деформаций от длины в осевом сечении внешней обоймы при числе роликов:

$$
\bigcirc-z=3 ; \triangle-z=4 ; \square-z=5
$$

ния деформаций подобен при любом числе роликов. Это дает возможность в дальнейшем оценивать только деформированное состояние цилиндрической оболочки и части ступицы, которые соответствуют длине $L=80 \mathrm{мм}$.

Анализ распределения деформации в радиальном сечении внешней обоймы в зависимости от полярного угла $\varphi$ (рис. 6) показывает, что внешняя обойма деформируется приблизительно симметрично относительно углов расположения роликов. Отрицательные величины указывают на то, что деформации цилиндрической оболочки направлены к ее центру.

Выбранное сечение соответствует зоне действия максимальных деформаций на свободном крае цилиндрической оболочки.

Как видно на рис. 6, при увеличении числа роликов происходит уменьшение радиальной деформации в 1,5-2,1 раза. Такая закономерность изменения деформаций объясняется теми же причинами, что и для напряжений, рассмотренными ранее. Сравнительный рас-

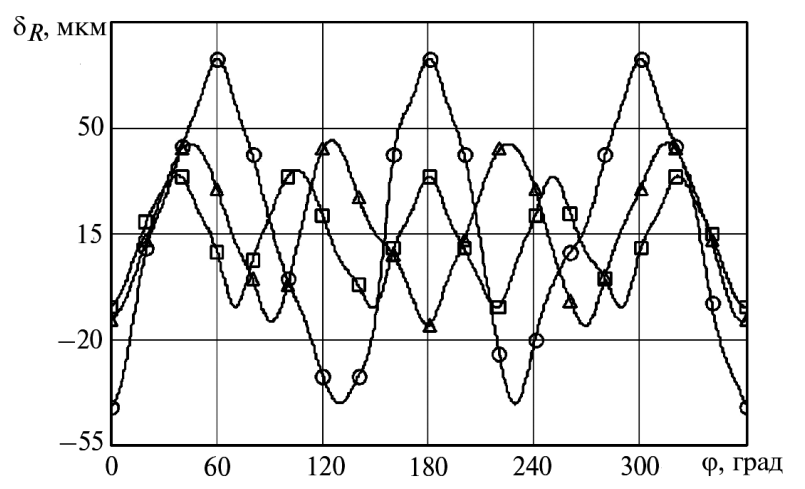

Puc. 6. Зависимость деформаций от полярного угла в радиальном сечении внешней обоймы при числе роликов:

$$
\bigcirc-z=3 ; \triangle-z=4 ; \square-z=5
$$


чет, проведенный по формуле работ [2], подтвердил выявленную закономерность. При этом расчетное значение деформации больше в 1,5-3,6 раз по сравнению с результатами конечно-элементного анализа. Это можно объяснить тем, что при проведении теоретического расчета отсутствует учет влияния кольцевой пластины на жесткость внешней обоймы.

\section{Выводы}

1. Численными расчетами в программе T-FLEX CAD 3D определены зоны действия и величины максимальных напряжений и деформаций во внешней обойме роликовых MCX и характер влияния на них основных геометрических параметров обоймы.

2. Установлено, что увеличение числа заклинивающихся роликов от 3 до 6 приводит к одновременному росту передаваемой роликовым MCX нагрузки и уменьшению возникающих во внешней обойме напряжений и деформаций.

3. Расчет внешней обоймы по методике, когда ее расчетная схема сводится к плоскому кольцу, нагруженному сосредоточенными силами, дает существенно завышенные значения напряжений и деформаций.

\section{Литература}

[1] Архангельский Г.В., Архангельский А.Г. Роликовые механизмы свободного хода. Одесса, Наука и техника, 2009, 92 с.

[2] Мальцев В.Ф. Роликовые механизмы свободного хода. Москва, Машиностроение, 1968, 415 с.

[3] Orthwein W.C. Clutches and brakes: design and selection. New York, Basel, Marcel Dekker, 2004, 330 p.

[4] Childs Peter R.N. Mechanical design. Oxford, ButterworthHeinemann, 2004, 358 p.

[5] Shigley Joseph E., Mischke Charles R. Standard handbook of machine design. New York, McGraw-Hill, 1996, 1761 p.
[6] Neale M.J. The tribology handbook. Oxford, ButterworthHeinemann, 2000, 298 p.

[7] Ряховский О.А., Иванов С.С. Справочник по муфтам. Ленинград, Политехника, 1991, 384 с.

[8] Хабрат Н.И. Анализ усилий в роликовой обгонной муфте. Тракторы и сельхозмашины, 2010, № 10, с. 53-56.

[9] Иванов М.Н., Финогенов В.А. Детали машин. Москва, Высшая школа, 2006, 408 с.

[10] Крупский В.И. Исследования и вопросы расчета механизмов свободного хода приводов стартеров ДВС. Дисс. ... канд. техн. наук. Одесса, ОТИПП им. М.В. Ломоносова, 1977,232 c.

[11] Леонов А.И. Микрохраповые механизмы свободного хода. Москва, Машиностроение, 1982. 219 с.

\section{References}

[1] Arkhangel'skii G.V., Arkhangel'skii A.G. Rolikovye mekhanizmy svobodnogo khoda [Roller Freewheel]. Odessa, Nauka i tekhnika publ., 2009. 92 p.

[2] Mal'tsev V.F. Rolikovye mekhanizmy svobodnogo khoda [Roller Freewheel]. Moscow, Mashinostroenie publ., 1968. 415 p.

[3] Orthwein W.C. Clutches and brakes: design and selection. New York, Basel, Marcel Dekker publ., 2004. 330 p.

[4] Childs Peter R.N. Mechanical design. Oxford, Butterworth-Heinemann publ., 2004. 358 p.

[5] Shigley Joseph E., Mischke Charles R. Standard handbook of machine design. New York, McGraw-Hill, 1996. $1761 \mathrm{p}$.

[6] Neale M.J. The tribology handbook. Oxford, Butterworth-Heinemann publ., 2000. 298 p.

[7] Riakhovskii O.A., Ivanov S.S. Spravochnik po muftam [Reference couplings]. Leningrad, Politekhnika publ., 1991. 384 p.

[8] Khabrat N.I. Analiz usilii v rolikovoi obgonnoi mufte [Analysis of effort in a roller overrunning clutch]. Traktory $i$ sel'khozmashiny [Tractors and farm machinery]. 2010, no. 10, pp. 53-56.

[9] Ivanov M.N., Finogenov V.A. Detali mashin [Machine Parts]. Moscow, Vysshaia shkola publ., 2006. 408 p.

[10] Krupskii V.I. Issledovaniia i voprosy rascheta mekhanizmov svobodnogo khoda privodov starterov DVS. Diss. kand. tekhn. nauk. [Research and problems of calculating freewheel drive engine starters. Cand. tehn. sci. diss.]. Odessa, OTIPP im. M.V. Lomonosova publ., 1977. 232 p.

[11] Leonov A.I. Mikrokhrapovye mekhanizmy svobodnogo khoda [Micro Ratchets freewheel]. Moscow, Mashinostroenie publ., 1982. 219 p.

Статья поступила в редакцию 11.06.2013

\section{Информация об авторах}

ЗОЛОТОВ Иван Анатольевич (Калининград) - аспирант кафедры «Теория механизмов и машин и детали машин». Калининградский государственный технический университет (236022, Калининград, Российская Федерация, Советский пр-т, д. 1, e-mail: zolivan@rambler.ru).

ШАРКОВ Олег Васильевич (Калининград) - доктор технических наук, доцент, профессор кафедры «Теория механизмов и машин и детали машин». Калининградский государственный технический университет (236022, Калининград, Российская Федерация, Советский пр-т, д. 1, e-mail: o_sharkov@mail.ru).

\section{Information about the authors}

ZOLOTOV Ivan Anatol'evich (Kaliningrad) - Post-Graduate of «Theory of Mechanisms and Machines and Machine Parts» Department. Kaliningrad State Technical University (KSTU, Sovetskiy pr., 1, 236022, Kaliningrad, Russian Federation, e-mail: zolivan@rambler.ru).

SHARKOV Oleg Vasil'evich (Kaliningrad) - Dr. Sc. (Eng.), Associate Professor, Professor of «Theory of Mechanisms and Machines and Machine Parts» Department. Kaliningrad State Technical University (KSTU, Sovetskiy pr., 1, 236022, Kaliningrad, Russian Federation, e-mail: o_sharkov@mail.ru). 